\title{
Terapia Nutricional e Adequação Calórico-Proteica em Pacientes Críticos com Lesão Renal Aguda
}

\author{
Luiza Pessoa de Araújo, ${ }^{1}$ Weverson Ferreira Lopes, ${ }^{1}$ Cláudia Sena de Pádua, ${ }^{2}$ \\ Patrícia Rezende do Prado, ${ }^{1}$ Thatiana Lameira Maciel Amaral ${ }^{3}$
}

\begin{abstract}
RESUMO
Este estudo tem o objetivo de descrever a terapia nutricional e a adequação calórico-proteica dos pacientes críticos que evoluíram com Lesão Renal Aguda (LRA). É um estudo observacional do tipo coorte prospectivo, realizado com pacientes em terapia enteral, admitidos em uma UTI pública. Foi considerada inadequação calórico-proteica os pacientes que não atingiram $80 \%$ do valor energético total em até 7 dias de internação e oferta de proteína $<1,5 \mathrm{~g} / \mathrm{kg}$ de peso $/$ dia. As diferenças entre os grupos foram obtidas pelo teste t-Student e qui-quadrado de Pearson. A inadequação energética e proteica, no 70 dia, foi observada nos pacientes do sexo masculino, com idade menor que 50 anos $(61,1 \%)$, que apresentavam comorbidades $(66,7 \%)$; a média de peso foi de $85,78 \pm 13,91 \mathrm{~kg}$; recebendo proteína $<1,5$ grama $(80,8 \%)$; manifestaram Lesão Renal Aguda $(55,6 \%)$; permaneceram internados por $15,72 \pm 10,80$ dias e evoluíram a óbito (83,3\%). Entre os pacientes com inadequação proteica, tanto no 3 ㅇ quanto no 70 dia, foram elevadas entre os pacientes que foram a óbito $(p<0,05)$. Na ocorrência de LRA, houve maior inadequação no valor energético $(28,6 \%)$, bem como maior incidência de intercorrências para a não infusão da dieta até o 3 o dia $(52,3 \%)$ quando comparados com os sem LRA $(p<0,05)$. A inadequação do suporte nutricional foi considerada alta nos pacientes críticos, e naqueles com LRA houve maior inadequação no valor energético. Ações de melhoria da qualidade da terapia nutricional devem ser realizadas para contribuir com desfechos favoráveis.
\end{abstract}

Palavras-chave: Lesão renal aguda. Terapia nutricional. Adequação calórico-proteica.

\section{NUTRITIONAL THERAPY AND CALORIC-PROTEIN ADEQUACY IN CRITICALLY} ILL PATIENTS WITH ACUTE KIDNEY INJURY

\section{ABSTRACT}

This study aims to describe the nutritional therapy and the caloric-protein adequacy of critically ill patients who developed acute kidney injury (AKI). It is an observational, prospective cohort study carried out with patients on enteral therapy, admitted to a public ICU. The caloric-protein inadequacy was considered to be patients who did not reach $80 \%$ of the total energy value within 07 days of hospitalization and protein supply $<1.5 \mathrm{~g} / \mathrm{kg}$ of weight/day. Differences between groups were obtained using the t-Student test and Pearson's chi-square test. Inadequate energy and protein, on the 7th day, was observed in male patients, aged less than 50 years $(61.1 \%)$, with comorbidities $(66.7 \%)$, the mean weight was $85.78 \pm 13.91 \mathrm{~kg}$, receiving protein $<1.5$ grams $(80.8 \%)$, manifested acute kidney injury (55.6\%), remained hospitalized for $15.72 \pm 10.80$ days and died (83.3\%). Among patients with inadequate protein, both on the 3rd and 7th day, they were high among patients who died ( $p$ $<0.05)$. In the occurrence of $A K I$, there was a greater inadequacy in the energy value $(28.6 \%)$, as well as a higher incidence of complications for the non-infusion of the diet until the 3rd day $(52.3 \%)$ when compared with those without AKI $(p<0.05)$. The inadequacy of nutritional support was considered high in the patients evaluated, and in those with AKI there was a greater inadequacy in the energy value. Actions to improve the quality of nutritional therapy must be carried out to contribute to favorable outcomes.

Keywords: Acute kidney injury. Nutritional therapy. Caloric-protein adequacy.

RECEBIDO EM: 4/9/2020

MODIFICAÇÕES SOLICITADAS EM: 7/9/2020

ACEITO EM: 23/10/2020

Universidade Federal do Acre. Rio Branco/AC, Brasil.

Secretaria do Estado de Saúde do Acre. Rio Branco/AC, Brasil.

Autora correspondente. Universidade Federal do Acre. Rodovia BR 364, Km 4 - Distrito Industrial, 69920-900, Rio Branco/AC, Brasil. http://lattes.cnpq. br/8598759724825005. http://orcid.org/0000-0002-9197-5633 thatianalameira27@gmail.com 


\section{INTRODUÇÃO}

A Lesão Renal Aguda (LRA) é definida como uma diminuição da função renal, sendo uma complicação grave que está associada a um aumento na morbimortalidade e apresenta uma alta incidência nas unidades de terapia intensiva (UTI) chegando a ocorrer em 50\% dos pacientes (MOHSENIN, 2017).

Segundo os critérios da Kidney Disease Improving Global Outcomes (KDIGO), a LRA pode ser definida como o aumento do nível da creatinina sérica acima de $0,3 \mathrm{mg} / \mathrm{dl}$ em 48 horas; ou o aumento da creatinina sérica 1,5 vezes em relação ao nível basal em 7 dias; ou a diurese menor que $0,5 \mathrm{ml} / \mathrm{kg} / \mathrm{h}$ durante 6 horas ("Kidney International Supplements", 2012; KIRSZTAJN et al., 2014; TEJERA et al., 2017).

Durante o tempo de evolução na UTI, a presença de modificações nas funções dos órgãos interfere na condição nutricional do paciente crítico, sendo a LRA, uma condição patológica, que afeta o metabolismo hídrico, eletrolítico e ácido-básico, além de interferir na transformação de todos os macronutrientes. Dentre as alterações cita-se a hiperglicemia, hipertrigliceridemia e o hipercatabolismo. $O$ efeito da associação dessas condições metabólicas, a imobilização do paciente e o suporte nutricional inadequado interferem diretamente na evolução clínica do paciente crítico (KELLUM, 2015; SINGER; COHEN, 2016).

A terapia nutricional (TN) deve ser aplicada adequadamente, objetivando promover a redução da morbimortalidade, diminuição da resposta catabólica, além de contribuir para um menor tempo de internação e, como consequência, redução no custo do tratamento. $O$ estado nutricional inadequado limita a eficácia do tratamento médico e a intervenção nutricional, pois resulta em sérias consequências para pacientes gravemente enfermos cujo estado de saúde já é comprometido, aumentando o risco de infecção, diminuindo a função renal, hepática e o funcionamento do sistema imunológico (TOLEDO; GONÇALVES; CASTRO, 2016; ZHANG; ZHANG; DONG, 2018)].

Nas últimas décadas, organizações e sociedades profissionais desenvolveram diretrizes direcionadas aos cuidados nutricionais do paciente crítico, no intuito de congregar evidências e subsidiar as decisões acerca da terapêutica para esses pacientes. A terapia nutricional adequada está inserida no plano terapêutico deste paciente, necessitando assim de uma abordagem pragmática que, ao mesmo tempo que leva em conta a complexidade de cada paciente, também proporciona uma abordagem uniforme e simples, que pode ser prontamente aplicada (MCCLAVE et al., 2016; SINGER; COHEN, 2016).

A adequação da oferta energética ao paciente crítico representa um importante desafio, sendo a terapia nutricional enteral (TNE), a primeira opção na impossibilidade de alimentação pela via oral, que frequentemente resulta em oferta insuficiente de energia. O início precoce da TNE deve ser evitado em pacientes com choque descontrolado, hipoxemia e acidose não controladas, sangramento gastrointestinal descontrolado, isquemia intestinal aberta (oclusiva ou não oclusiva), obstrução intestinal, síndrome do compartimento abdominal, volume de aspirado gástrico $>500 \mathrm{ml} / 6 \mathrm{~h}$ ou fístula de alto débito se o acesso à alimentação distal confiável não for possível (REINTAM BLASER et al., 2017).

No que diz respeito ao início precoce de nutrição enteral (NE), as diretrizes preconizam que, se o tubo digestivo estiver viável e o paciente hemodinamicamente estável, a NE deve ser iniciada dentro de 24 a 48 horas (SINGER et al., 2019). A Sociedade Europeia de Medicina Intensiva (ESICM), endossada pelo Grupo de Trabalho sobre Função Gastrointestinal na Seção de Metabolismo, Endocrinologia e Nutrição (MEN), também fornece diretrizes baseadas em evidências para nutrição enteral precoce em pacientes críticos, concentrando-se em condições clínicas específicas frequentemente associadas a atraso na nutrição enteral (REINTAM BLASER et al., 2017). O estabelecimento da TN adequada permitirá que sejam atingidas as necessidades energético-proteicas, levando, possivelmente, a um melhor resultado clínico (MCCLAVE et al., 2016; PATEL et al., 2017; SINGER; COHEN, 2016).

Quando aplicada adequadamente, a terapia nutricional fornecida a pacientes críticos pode resultar em redução da mortalidade e infecções, além de melhorar a qualidade de vida relacionada à saúde. No entanto, existe o potencial de causar danos, principalmente se usado de forma inadequada ou com a população de pacientes errada (DHALIWAL et al., 2014).

Com relação à terapia nutricional dos pacientes com LRA, visualiza-se uma dificuldade no manejo nutricional adequado, pois os pacientes críticos necessitam de um aporte proteico elevado, muito embora, no paciente que desenvolve LRA a oferta proteica deve ser planejada de uma forma mais criteriosa, para que contemple não só as necessidades nutricionais do paciente, mas que também possa reduzir as complicações da LRA (BERBEL et al., 2014; TEJERA et al., 2017; TOLEDO; GONÇALVES; CASTRO, 2016). 
Diante da complexidade e da alta incidência de LRA na UTI, este trabalho objetiva descrever a terapia nutricional e a adequação calórico-proteica segundo as diretrizes de nutrição enteral, dos pacientes críticos que evoluíram com lesão renal aguda, em uma Unidade de Terapia Intensiva, de Rio Branco, Acre.

\section{MATERIAIS E MÉTODO}

Trata-se de um estudo observacional do tipo caso-coorte, com abordagem quantitativa, em uma Unidade de Terapia Intensiva, de um hospital público de Urgência e Emergência, de Rio Branco, Acre. Os pacientes estudados foram todos aqueles admitidos na unidade, com idade maior que 18 anos utilizando terapia nutricional enteral exclusiva. Foram excluídos pacientes com diagnóstico prévio de insuficiência renal crônica, transplantados renais, pacientes admitidos com morte encefálica, internações com duração menor que 24 horas ou iminente risco de morte dentro das primeiras 48 horas de internação, gestantes, puérperas, indígenas e estrangeiros. A pesquisa teve duração de 04 meses, de maio a agosto de 2019.

Dos 130 pacientes internados na UTI no período de coleta, 28 foram excluídos por não atenderem os critérios da pesquisa, dessa forma a presente amostra incluiu 102 pacientes (78\% dos potenciais participantes).

Foi definido LRA como o aumento da creatinina em 1,5 a 1,9 vezes o valor basal em 7 dias ou aumento da creatinina sérica $>0,3 \mathrm{mg} / \mathrm{dl}$ em $48 \mathrm{~h}$. Assim como, volume urinário menor que $0,5 \mathrm{ml} / \mathrm{Kg} / \mathrm{h}$ por $6-12 \mathrm{~h}$. Para creatinina basal foi considerado o valor obtido no momento da admissão na UTI, semelhante a outros estudos que trabalharam essa temática (HOSTE et al., 2015; KIRSZTAJN et al., 2014).

As diretrizes recomendam que a nutrição enteral (NE) deve ser iniciada no período de 24 a 48 horas, desde que o paciente esteja hemodinamicamente estável e com o tubo digestivo íntegro para receber o suporte de NE (MCCLAVE et al., 2016; REINTAM BLASER et al., 2017; SINGER et al., 2019).

Para estimar a necessidade energética de pacientes com LRA, foram utilizados os Guidelines Enteral Nutrition da Sociedade Europeia de Nutrição Enteral e Parenteral (ESPEN, European Society for Parenteral and Enteral Nutrition, 2019) e os parâmetros adotados pela Sociedade Americana de Nutrição Enteral e Parenteral (ASPEN, American Society for Parenteral and Enteral Nutrition, 2016) (MCCLAVE et al., 2016; SINGER et al., 2019).
O peso do paciente foi identificado através da balança, incorporada na cama do leito, da marca Hospimetal $^{\circledR}$, modelo Cama Fawler HM. 2002E, 4 motores, com capacidade máxima de $250 \mathrm{~kg}$. O gasto energético foi estimado por equações preditivas, utilizando as recomendações, da ASPEN e ESPEN, 25-30kcal/kg de peso/dia (SINGER et al., 2019). A recomendação de proteína superior a $1,5 \mathrm{~g} / \mathrm{kg} /$ dia objetiva minimizar as perdas nitrogenadas. Pacientes em terapia extracorpórea, com hipercatabolismo moderado, a recomendação é de 1,0 a 1,5 g/ kg/dia, em hemodiálise contínua, com hipercatabolismo grave, a oferta proteica pode atingir até $2,5 \mathrm{~g} / \mathrm{kg} /$ dia (MCCLAVE et al., 2016; PATEL et al., 2017).

No período de 48 a 72 horas de internação o suporte de NE deve atingir 50 a $65 \%$ das necessidades calóricas e $100 \%$ até sete dias. A data de início da dieta enteral e a evolução de calorias e proteínas foram registradas e comparadas com as diretrizes no terceiro dia (D3) de internação na UTI, compreendendo $50 \%$ das necessidades e no sétimo dia (D7), compreendendo $100 \%$ das necessidades (MCCLAVE et al., 2016). Foi considerado início precoce de NE quando esta ocorreu dentro de 48 horas de internação. Para o cálculo dessa adequação, foi multiplicada a quantidade de quilocalorias fornecida pela dieta, multiplicado por 100 e dividido pelo valor energético total que o paciente precisa.

As informações foram coletadas por meio de uma ficha de coleta de dados que continha informações gerais sobre a admissão como data, nome do paciente, idade, sexo, profissão, escolaridade, região de moradia (zona rural ou urbana). Para avaliar a evolução terapêutica nutricional, além de considerar as recomendações sobre a adequação do valor energético total (VET), também levou em consideração as intercorrências que interferiam na infusão da dieta, como instabilidade hemodinâmica, procedimentos (exames, cirúrgicos), extubação, intubação, uso de ventilação não invasiva (VNI), êmese, sonda nasogástrica aberta (débito pela sonda), retirada da sonda, distensão abdominal, constipação e diarreia.

$\mathrm{Na}$ descrição das variáveis contínuas foram apresentadas medidas de tendência central (média e desvio-padrão) e as variáveis categóricas foram expressas por distribuição de frequências absoluta e relativa. Na comparação de grupos foram realizados os testes t de Student e o teste qui-quadrado de Pearson ou teste exato de Fisher, considerando a natureza das variáveis contínuas e categóricas, respectivamente. 
Em todas as análises, adotou-se o nível de significância de $\alpha=5 \%$. Os dados foram analisados com o programa SPSS ${ }^{\circledR}$, versão 20.0 .

Este estudo foi aprovado pelo Comitê de Ética em Pesquisa da Universidade Federal do Acre sob parecer $n$ ㅇ 3.294 .722 e obedeceu a resolução $n$ ‥ $466 / 2012$.

\section{RESULTADOS}

Os pacientes críticos eram predominantemente do sexo masculino $(73,5 \%)$ e com idade menor que 50 anos $(55,9 \%)$. O tempo médio de internação foi de $16,93 \pm 14,41$ dias, sendo que a maioria morava na zona urbana $(81,4 \%)$, com ocupação $(61,8 \%)$ e estudaram até o ensino fundamental $(55,3 \%)$, internados devido a trauma (33,3\%), possuíam comorbidades $(54,9 \%)$, estavam utilizando drogas vasoativas $(52,9 \%)$ e em ventilação mecânica (64,7\%). Com relação ao tipo de dieta na admissão estavam em dieta zero (55,9\%) (Tabela 1).

Tabela 1 - Caracterização dos Pacientes admitidos em uma Unidade de Terapia Intensiva de Rio Branco, Acre, Brasil, 2019

\begin{tabular}{|c|c|c|}
\hline Variáveis & $\mathbf{N}$ & $\%$ \\
\hline Idade $^{\text {a }}$ & $46,79 \pm 17,66$ & - \\
\hline Tempo de internação ${ }^{b}$ & $16,93 \pm 14,41$ & - \\
\hline \multicolumn{3}{|l|}{ Faixa etária ${ }^{c}$} \\
\hline$<50$ & 57 & 55,9 \\
\hline$\geq 50$ & 45 & 44,1 \\
\hline \multicolumn{3}{|l|}{ Sexo } \\
\hline Masculino & 75 & 73,5 \\
\hline Feminino & 27 & 26,5 \\
\hline \multicolumn{3}{|l|}{ Local de Residência } \\
\hline Urbana & 79 & 81,4 \\
\hline Rural & 18 & 18,6 \\
\hline \multicolumn{3}{|l|}{ Ocupação } \\
\hline Sim & 63 & 61,8 \\
\hline Não & 39 & 38,2 \\
\hline \multicolumn{3}{|l|}{ Escolaridade } \\
\hline Não Alfabetizado & 10 & 10,5 \\
\hline Até o Ensino Fundamental & 43 & 45,3 \\
\hline Até o Ensino Médio & 38 & 40,0 \\
\hline Ensino Superior & 04 & 04,2 \\
\hline \multicolumn{3}{|l|}{ Procedência } \\
\hline Centro Cirúrgico & 39 & 38,2 \\
\hline Clínica & 42 & 41,2 \\
\hline Outros & 21 & 20,6 \\
\hline \multicolumn{3}{|l|}{ Motivo da internação } \\
\hline Trauma & 34 & 33,3 \\
\hline PO Abdominal/Neurológico & 08 & 07,8 \\
\hline Sepse & 22 & 21,6 \\
\hline Cardíaco & 12 & 11,8 \\
\hline
\end{tabular}

\begin{tabular}{lcc}
$\begin{array}{l}\text { Outras causas } \\
\text { Comorbidades } \\
\text { Sim }\end{array}$ & 26 & 25,5 \\
$\quad$ Não & 56 & 54,9 \\
Drogas vasoativas & 46 & 45,1 \\
$\quad$ Sim & 54 & 52,9 \\
$\quad$ Não & 48 & 47,1 \\
Oxigenioterapia & & \\
$\quad$ Ventilação Mecânica & 66 & 64,7 \\
$\quad$ Ar Ambiente & 29 & 28,4 \\
$\quad$ Outro tipo & 07 & 06,9 \\
Prescrição de dieta da admissão & & \\
$\quad$ Zero & 57 & 55,9 \\
$\quad$ Via Oral & 15 & 14,7 \\
$\quad$ Nutrição Enteral & 30 & 29,4 \\
\hline TOTAL & 102 & 100,0 \\
\hline
\end{tabular}

*PO = pós operatório. aldade em anos (Média \pm Desvio Padrão). ${ }^{\text {bDias de }}$ internação. Faixa etária em anos.

Dos pacientes que tiveram inadequação energética e proteica, a média de idade foi de $50,83 \pm$ 16,52 , eram do sexo masculino, apresentavam comorbidades, utilizavam drogas vasoativas e estavam em ventilação mecânica. Com relação ao peso dos pacientes que não tiveram adequação energética e proteica no 70 dia de internação a média foi de $85,78 \pm 13,91 \mathrm{~kg}$, a maioria não atingiu a meta proteica, permaneceram internados por 16 dias, em média, manifestaram lesão renal aguda e evoluíram a óbito. Houve diferença estatística entre os grupos de adequação e inadequação energética e proteica para idade, peso, aporte proteico e tempo de permanência na UTI $(p<0,05)$ (Tabela 2).

Entretanto, os pacientes que tiveram nutrição precoce eram mais jovens, com idade inferior a 50 anos e do sexo masculino. Também possuíam comorbidades, utilizavam drogas vasoativas e estavam em ventilação mecânica. Em relação ao peso, a média foi de $77,4 \pm 16,7 \mathrm{~kg}$. Apesar do início precoce da terapia nutricional, estes pacientes também não atingiram a meta proteica, a média de tempo de permanência na UTI foi de aproximadamente 17 dias, não manifestaram lesão renal aguda, entretanto o desfecho, na maioria dos casos, também foi o óbito. No entanto, não houve diferença entre os grupos com e sem nutrição precoce nas variáveis pesquisadas $(p>0,05)$. 
Tabela 2 - Descrição da adequação do valor energético-proteico até o 7o dia de internação e nutrição precoce em uma Unidade de Terapia Intensiva de Rio Branco, Acre, Brasil, 2019

\begin{tabular}{|c|c|c|c|c|c|c|}
\hline \multirow[t]{3}{*}{ Variáveis } & \multicolumn{2}{|c|}{ Adequação Energética no 7ㅇ dia } & \multirow{3}{*}{ p-valor ${ }^{a}$} & \multicolumn{2}{|c|}{ Nutrição Precoce } & \multirow{3}{*}{ p-valor ${ }^{a}$} \\
\hline & Sim & Não & & Sim & Não & \\
\hline & $\mathrm{N}(\%)$ & $\mathrm{N}(\%)$ & & $\mathrm{N}(\%)$ & $\mathrm{N}(\%)$ & \\
\hline Idade $^{c}$ & $45,15 \pm 18,22$ & $50,83 \pm 16,52$ & $<0,001$ & $\begin{array}{r}46,73 \pm \\
18,04\end{array}$ & $47,36 \pm 14,85$ & 0,210 \\
\hline$<50$ & $32(60,4)$ & $07(38,9)$ & \multirow{2}{*}{0,113} & $50(54,9)$ & $07(63,6)$ & \multirow{2}{*}{0,415} \\
\hline$\geq 50$ & $21(39,6)$ & $11(61,1)$ & & $41(45,1)$ & $04(36,4)$ & \\
\hline \multicolumn{7}{|l|}{ Sexo } \\
\hline Masculino & $41(77,4)$ & $11(61,1)$ & \multirow{2}{*}{0,179} & $65(71,4)$ & $10(90,9)$ & \multirow{2}{*}{0,153} \\
\hline Feminino & $12(22,6)$ & $07(38,9)$ & & $26(28,6)$ & $01(9,1)$ & \\
\hline \multicolumn{7}{|l|}{ Motivo da internação } \\
\hline Sepse & $13(24,5)$ & $04(23,5)$ & \multirow{3}{*}{0,568} & $20(22,2)$ & $02(18,2)$ & \multirow{3}{*}{0,287} \\
\hline Trauma & $19(35,8)$ & $04(23,5)$ & & $28(31,1)$ & $06(54,5)$ & \\
\hline Outros & $21(39,6)$ & $09(52,9)$ & & $42(46,7)$ & $03(27,3)$ & \\
\hline \multicolumn{7}{|l|}{ Comorbidades } \\
\hline Sim & $26(49,1)$ & $12(66,7)$ & \multirow[t]{2}{*}{0,196} & $52(57,1)$ & $04(36,4)$ & \multirow[t]{2}{*}{0,162} \\
\hline Não & $27(50,9)$ & $06(33,3)$ & & $39(42,9)$ & $07(63,6)$ & \\
\hline \multicolumn{7}{|l|}{ Drogas vasoativas } \\
\hline Sim & $33(62,3)$ & $12(66,7)$ & \multirow[t]{2}{*}{0,738} & $47(51,6)$ & $07(63,6)$ & \multirow[t]{2}{*}{0,335} \\
\hline Não & $20(37,7)$ & $06(33,3)$ & & $44(48,4)$ & $04(36,4)$ & \\
\hline \multicolumn{7}{|l|}{ Ventilação mecânica } \\
\hline Sim & $42(79,2)$ & $15(83,3)$ & \multirow[t]{2}{*}{0,501} & $58(63,7)$ & $08(72,7)$ & \multirow{2}{*}{0,410} \\
\hline Não & $11(20,8)$ & $03(16,7)$ & & $33(36,3)$ & $03(27,3)$ & \\
\hline Peso (média $\pm D P$ ) & $70,55 \pm 15,57$ & $85,78 \pm 13,91$ & $<0,001$ & $73,4 \pm 15,9$ & $77,4 \pm 16,7$ & 0,428 \\
\hline \multicolumn{7}{|l|}{ Proteína (g/kg/dia) } \\
\hline$<1,5$ & $11(40,7)$ & $21(80,8)$ & \multirow[t]{2}{*}{0,003} & $42(60,0)$ & $06(100,0)$ & 0,056 \\
\hline$\geq 1,5$ & $16(59,3)$ & $05(19,2)$ & & $28(40,0)$ & $00(0,0)$ & \\
\hline Evolução & & & & & & \\
\hline Alta & $17(32,7)$ & $03(16,7)$ & 0,195 & $28(31,1)$ & $03(27,3)$ & 0,548 \\
\hline Óbito & $35(67,3)$ & $15(83,3)$ & & $62(68,9)$ & $08(72,7)$ & \\
\hline Tempo de permanência na UTI & $21,58 \pm 16,12$ & $15,72 \pm 10,80$ & $<0,001$ & $17,1 \pm 14,5$ & $15,2 \pm 14,6$ & 0,673 \\
\hline Lesão Renal Aguda & & & & & & \\
\hline Sim & $26(49,1)$ & $10(55,6)$ & 0,634 & $37(40,7)$ & $07(63,6)$ & 0,129 \\
\hline Não & $27(50,9)$ & $08(44,4)$ & & $54(59,3)$ & $04(36,4)$ & \\
\hline Total & $27(50,9)$ & $26(49,1)$ & & $91(89,2)$ & $11(10,8)$ & $102(100,0)$ \\
\hline
\end{tabular}

${ }^{a}$ Qui-quadrado de Pearson e ${ }^{b}$ Teste exato de Fisher; DP=desvio-padrão. Idade em anos (média $\left.\pm D P\right)$. ${ }^{d}$ média $\pm D P$.

Entre os pacientes que manifestaram LRA e foram a óbito eram na maioria do sexo masculino, tinham idade menor que 50 anos, sendo procedentes do centro cirúrgico, internados devido ao trauma, permanecendo na unidade por mais de 15 dias. Esses pacientes, em sua maioria, apresentavam comorbidades, estavam em ventilação mecânica, sendo ventilados a uma pressão positiva expiratória final (PEEP) maior que 10 , utilizavam drogas vasoativas, tiveram a aferição de glicemia sérica menor que $180 \mathrm{mg} / \mathrm{dl}$ e estavam sob dieta zero. Houve diferença estatística quanto ao sexo na ocorrência de LRA $(p<0,05)$.

A Tabela 4 trata sobre as condições nutricionais segundo os fatores prognósticos do óbito e de lesão renal aguda. Entre os pacientes com inadequação proteica, tanto no 3 ㅇ quanto no 70 dia, foram eleva- 
Tabela 3 - Características clínicas e demográficas segundo desfecho e presença de lesão renal dos pacientes admitidos em uma Unidade de Terapia Intensiva de Rio Branco, Acre, Brasil, 2019

\begin{tabular}{|c|c|c|c|c|c|c|c|}
\hline \multirow[t]{2}{*}{ Variáveis } & \multirow[t]{2}{*}{ Total } & \multicolumn{2}{|c|}{ LRA } & \multirow[t]{2}{*}{ p-valor ${ }^{a}$} & \multicolumn{2}{|c|}{ Desfecho } & \multirow[t]{2}{*}{ p-valor } \\
\hline & & Sim & Não & & Alta & Óbito & \\
\hline \multicolumn{8}{|l|}{ IDADE $^{\mathrm{c}}$} \\
\hline$<50$ & $57(55,9)$ & $34(58,6)$ & $23(52,3)$ & \multirow[t]{2}{*}{0,523} & $19(61,3)$ & $37(52,9)$ & \multirow[t]{2}{*}{0,432} \\
\hline$\geq 50$ & $45(44,1)$ & $24(41,4)$ & $21(47,7)$ & & $12(38,7)$ & $33(47,1)$ & \\
\hline \multicolumn{8}{|l|}{ Sexo } \\
\hline Feminino & $27(26,5)$ & $20(34,5)$ & $07(15,9)$ & \multirow[t]{2}{*}{0,035} & $11(35,5)$ & $16(22,9)$ & \multirow[t]{2}{*}{0,186} \\
\hline Masculino & $75(73,5)$ & $38(65,5)$ & $37(84,1)$ & & $20(64,5)$ & $54(77,1)$ & \\
\hline \multicolumn{8}{|c|}{ Tempo de Permanência na UTI ${ }^{d}$} \\
\hline Até 7 & $28(27,5)$ & $20(34,5)$ & $08(18,2)$ & \multirow[t]{3}{*}{0,310} & $10(32,3)$ & $18(25,7)$ & \multirow[t]{3}{*}{0,262} \\
\hline $8-14$ & $35(34,3)$ & $22(37,9)$ & $13(29,5)$ & & $13(41,9)$ & $22(31,4)$ & \\
\hline 15 e mais & $39(38,2)$ & $16(27,6)$ & $23(52,3)$ & & $08(25,8)$ & $30(42,9)$ & \\
\hline \multicolumn{8}{|l|}{ Procedência } \\
\hline Clínica & $42(41,2)$ & $26(44,8)$ & $16(36,4)$ & \multirow[t]{3}{*}{0,628} & $15(48,4)$ & $27(38,6)$ & \multirow[b]{3}{*}{0,098} \\
\hline Cirúrgica & $39(38,2)$ & $20(34,5)$ & $19(43,2)$ & & $07(22,6)$ & $31(44,3)$ & \\
\hline Outras & $21(20,6)$ & $12(20,7)$ & $09(20,5)$ & & $09(29,0)$ & $12(17,1)$ & \\
\hline \multicolumn{8}{|c|}{ Motivo da Internação } \\
\hline Sepse & $22(21,6)$ & $12(20,7)$ & $10(22,7)$ & \multirow{5}{*}{0,911} & $06(19,4)$ & $16(22,9)$ & \\
\hline Trauma & $34(33,3)$ & $21(36,2)$ & $13(29,5)$ & & $11(35,5)$ & $22(31,4)$ & \\
\hline $\begin{array}{l}\text { PO Abd. / } \\
\text { Neuro }\end{array}$ & $08(7,8)$ & $05(8,6)$ & $03(6,8)$ & & $02(6,5)$ & $06(8,6)$ & \multirow{3}{*}{0,983} \\
\hline Cardíaco & $12(11,8)$ & $07(12,1)$ & $05(11,4)$ & & $04(12,9)$ & $08(11,4)$ & \\
\hline Outras causas & $26(25,5)$ & $13(22,4)$ & $13(29,5)$ & & $08(25,8)$ & $18(25,7)$ & \\
\hline \multicolumn{8}{|l|}{ Comorbidades } \\
\hline Não & $46(45,1)$ & $27(46,6)$ & $19(43,2)$ & \multirow[t]{2}{*}{0,735} & $17(54,8)$ & $28(40,0)$ & \multirow[b]{2}{*}{0,166} \\
\hline Sim & $56(54,9)$ & $31(53,4)$ & $25(56,8)$ & & $14(45,2)$ & $42(60,0)$ & \\
\hline Ventilação Mecâ & & & & & & & \\
\hline Não & $36(35,3)$ & $22(37,9)$ & $14(31,8)$ & 0,522 & $12(38,7)$ & $24(34,3)$ & \\
\hline Sim & $66(64,7)$ & $36(62,1)$ & $30(68,2)$ & & $19(61,3)$ & $46(65,7)$ & 0,669 \\
\hline PEEP* & & & & & & & \\
\hline 6 a 9 & $47(71,2)$ & $27(75,0)$ & $20(66,7)$ & 0,457 & $13(68,4)$ & $33(71,7)$ & 0,789 \\
\hline 10 e mais & $19(28,8)$ & $09(25,0)$ & $10(33,3)$ & & $06(31,6)$ & $13(28,3)$ & \\
\hline Drogas Vasoativa & & & & & & & \\
\hline Não & $48(47,1)$ & $32(55,2)$ & $16(36,4)$ & 0,059 & $17(54,8)$ & $31(44,3)$ & \\
\hline Sim & $54(52,9)$ & $26(44,8)$ & $28(63,6)$ & & $14(45,2)$ & $39(55,7)$ & 0,327 \\
\hline Glicemia Séricae & & & & & & & \\
\hline$\leq 180$ & $72(70,6)$ & $44(75,9)$ & $28(63,6)$ & 0,143 & $19(61,3)$ & $52(74,3)$ & \\
\hline 180- 299 & $22(21,6)$ & $12(20,7)$ & $10(22,7)$ & & $09(29,0)$ & $13(18,6)$ & 47 \\
\hline$\geq 300$ & $08(07,8)$ & $02(03,4)$ & $06(13,6)$ & & $03(09,7)$ & $05(07,1)$ & 0,413 \\
\hline Prescrição Nutric & & & & & & & \\
\hline Zero & $57(55,9)$ & $31(53,4)$ & $26(59,1)$ & 0,694 & $14(45,2)$ & $42(60,0)$ & \\
\hline Via Oral & $15(14,7)$ & $10(17,2)$ & $05(11,4)$ & & $07(22,6)$ & $08(11,4)$ & \\
\hline Enteral & $30(29,4)$ & $17(29,3)$ & $13(29,5)$ & & $10(32,3)$ & $20(28,6)$ & 0,253 \\
\hline Total & & $44(43,1)$ & $58(56,9)$ & & $95(93,1)$ & $07(6,9)$ & \\
\hline
\end{tabular}

${ }^{a}$ Qui-quadrado de Pearson e ${ }^{b}$ Teste exato de Fisher; DP = desvio-padrão, PEEP = pressão positiva expiratória final. ${ }^{\mathrm{C}}$ Idade em anos. ${ }^{\mathrm{d}}$ Dias de internação. ${ }^{\mathrm{e}} \mathrm{mg} / \mathrm{dL}$.

das entre os pacientes que foram a óbito $(p<0,05)$. Observou-se adequação calórica, no terceiro dia, em $90,9 \%$ dos pacientes que não evoluíram com LRA, com significância estatística. Na ocorrência de LRA, houve maior inadequação no valor energético $(28,6 \%)$, bem como maior incidência de intercorrências para a não infusão da dieta até o 3 o dia (52,3\%) quando comparado com os sem LRA (Tabela 4). 
Tabela 4 - Condição nutricional segundo fatores prognósticos do óbito e de lesão renal aguda entre os pacientes em uma unidade de terapia intensiva segundo de Rio Branco, Acre, Brasil, 2019

\begin{tabular}{|c|c|c|c|c|}
\hline Desfecho & Alta & Óbito & Total & p-valor ${ }^{a}$ \\
\hline Adequação de proteína no 3ำ dia de internaçãoc & & & & 0,001 \\
\hline$<1,5$ & $11(55,0)$ & $45(90,0)$ & $56(80,0)$ & \\
\hline$\geq 1,5$ & $09(45,0)$ & $05(10,0)$ & $14(20,0)$ & \\
\hline Adequação de proteína no 70 dia de internação ${ }^{c}(\mathrm{~g} / \mathrm{kg} / \mathrm{c}$ & & & & 0,017 \\
\hline$<1,5$ & $09(42,9)$ & $39(72,2)$ & $48(64,0)$ & \\
\hline$\geq 1,5$ & $12(57,1)$ & $15(27,8)$ & $27(36,0)$ & \\
\hline Adequação do valor energético total no 3 dia & & & & 0,302 \\
\hline$<80 \%$ & $06(15,0)$ & $07(25,0)$ & $13(19,1)$ & \\
\hline$\geq 80 \%$ & $34(85,0)$ & $21(75,0)$ & $55(80,9)$ & \\
\hline Adequação do valor energético total no 70 dia & & & & 0,685 \\
\hline$<80 \%$ & $13(46,4)$ & $13(52,0)$ & $26(49,1)$ & \\
\hline$\geq 80 \%$ & $15(53,6)$ & $12(48,0)$ & $27(50,9)$ & \\
\hline Intercorrências até o 3ㅇ dia de internação & & & & 0,962 \\
\hline Não & $18(58,1)$ & $41(58,6)$ & $59(58,4)$ & \\
\hline Sim & $13(41,9)$ & $29(41,4)$ & $42(41,6)$ & \\
\hline Intercorrências até o 70 dia de internação & & & & 0,752 \\
\hline Não & $14(45,2)$ & $34(48,6)$ & $48(47,5)$ & \\
\hline Sim & $17(54,8)$ & $36(51,4)$ & $53(52,5)$ & \\
\hline Suspensão da dieta até o 3ำ dia de internação & & & & 0,115 \\
\hline Não & $12(38,7)$ & $39(55,7)$ & $51(50,5)$ & \\
\hline Sim & $19(61,3)$ & $31(44,3)$ & $50(49,5)$ & \\
\hline Suspensão da dieta até o 70 dia de internação & & & & 0,315 \\
\hline Não & $10(32,3)$ & $30(42,9)$ & $40(39,6)$ & \\
\hline Sim & $21(67,7)$ & $40(57,1)$ & $61(60,4)$ & \\
\hline Glicemia sérica até o 30 dia de internação ${ }^{d}$ & & & & 0,520 \\
\hline$<180$ & $20(64,5)$ & $40(57,1)$ & $60(59,4)$ & \\
\hline $\begin{array}{l}180-299 \\
\geq 300\end{array}$ & $\begin{array}{l}09(29,0) \\
02(06,5)\end{array}$ & $\begin{array}{l}20(28,6) \\
10(14,3)\end{array}$ & $\begin{array}{l}29(28,7) \\
12(11,9)\end{array}$ & \\
\hline \multicolumn{5}{|l|}{ Glicemia sérica até o 7ºdia de internação ${ }^{d}$} \\
\hline$<180$ & $16(51,6)$ & $39(55,7)$ & $55(54,5)$ & 0,590 \\
\hline 180- 299 & $07(22,6)$ & $19(27,1)$ & $26(25,7)$ & \\
\hline$\geq 300$ & $08(25,8)$ & $12(17,1)$ & $20(19,8)$ & \\
\hline Nutrição Precoce & & & & 0,548 \\
\hline Sim & $28(90,3)$ & $62(88,6)$ & $90(89,1)$ & \\
\hline Não & $03(9,7)$ & $08(11,4)$ & $11(10,9)$ & \\
\hline Lesão Renal Aguda & Não & Sim & Total & p-valor ${ }^{a}$ \\
\hline Adequação de proteína até o 3ㅇ diac & & & & 0,725 \\
\hline$<1,5$ & $27(77,1)$ & $29(80,6)$ & $56(78,9)$ & \\
\hline$\geq 1,5$ & $08(22,9)$ & $07(19,4)$ & $15(21,1)$ & \\
\hline Adequação de proteína até o 70 diac & & & & 0,109 \\
\hline$<1,5$ & $20(54,1)$ & $28(71,8)$ & $48(63,2)$ & \\
\hline$\geq 1,5$ & $17(45,9)$ & $11(28,2)$ & $28(36,8)$ & \\
\hline Adequação do valor energético total no 3 dia & & & & 0,041 \\
\hline$<80 \%$ & $03(09,1)$ & $10(28,6)$ & $13(19,1)$ & \\
\hline$\geq 80 \%$ & $30(90,9)$ & $25(71,4)$ & $55(80,9)$ & \\
\hline Adequação do valor energético total no 70 dia & & & & 0,328 \\
\hline$<80 \%$ & $10(41,7)$ & $16(55,2)$ & $26(49,1)$ & \\
\hline$\geq 80 \%$ & $14(58,3)$ & $13(44,8)$ & $27(50,9)$ & \\
\hline Intercorrências para a não infusão da dieta até o 3 dia & & & & 0,047 \\
\hline Não & $39(67,2)$ & $21(47,7)$ & $60(58,8)$ & \\
\hline Sim & $19(32,8)$ & $23(52,3)$ & $42(41,2)$ & \\
\hline Intercorrências para a não infusão da dieta até o 70 dia & & & & 0,906 \\
\hline Não & $27(46,6)$ & $21(47,7)$ & $48(47,1)$ & \\
\hline Sim & $31(53,4)$ & $23(52,3)$ & $54(52,9)$ & \\
\hline Suspensão da dieta até o 3ำ dia de internação & & & & 0,530 \\
\hline Não & $28(48,3)$ & $24(54,5)$ & $52(51,0)$ & \\
\hline Sim & $30(51,7)$ & $20(45,5)$ & $50(49,0)$ & \\
\hline
\end{tabular}


Suspensão da dieta até o 70 dia de internação

\begin{tabular}{|c|c|c|c|c|}
\hline & & & & \\
\hline $\begin{array}{l}\text { Não } \\
\text { Sim }\end{array}$ & $22(37,9)$ & $18(40,9)$ & $40(39,2)$ & \\
\hline Glicemia sérica até o 3 dia de internaçãod & & & & 0,420 \\
\hline $\begin{array}{l}<180 \\
180-299\end{array}$ & $\begin{array}{l}35(60,3) \\
14(24,1)\end{array}$ & $\begin{array}{l}25(56,8) \\
15(34,1)\end{array}$ & $\begin{array}{l}60(58,8) \\
29(28,4)\end{array}$ & \\
\hline$>300$ & $09(15,5)$ & $04(09,1)$ & $13(12,7)$ & \\
\hline Glicemia sérica até o 70 dia de internação ${ }^{d}$ & & & & 0,606 \\
\hline$<180$ & $32(55,2)$ & $23(52,3)$ & $55(53,9)$ & \\
\hline $\begin{array}{l}180-299 \\
>300\end{array}$ & $\begin{array}{l}16(27,6) \\
10(17,2)\end{array}$ & $\begin{array}{l}10(22,7) \\
11(25,0)\end{array}$ & $\begin{array}{l}26(25,5) \\
21(20,6)\end{array}$ & \\
\hline Nutrição precoce & & & & \\
\hline $\begin{array}{l}\text { Sim } \\
\text { Não }\end{array}$ & $\begin{array}{l}54(93,1) \\
04(06,9)\end{array}$ & $\begin{array}{l}37(84,1) \\
07(15,9)\end{array}$ & $\begin{array}{l}91(89,2) \\
11(10,8)\end{array}$ & 0,146 \\
\hline
\end{tabular}

${ }^{a}$ Qui-quadrado de Pearson e ${ }^{\mathrm{b}}$ Teste exato de Fisher. ${ }^{\mathrm{c}} \mathrm{g} / \mathrm{kg} / \mathrm{dia} .{ }^{\mathrm{d}} \mathrm{mg} / \mathrm{dL}$.

\section{DISCUSSÃO}

O perfil do paciente grave, favorece a elevada incidência de óbitos, isto devido a diversas variáveis que influenciam diretamente no prognóstico, sendo a TN um coadjuvante no tratamento do paciente (PEREIRA; WADY; VELARDE, 2016). Em um estudo realizado na UTI de um hospital universitário do Rio de Janeiro, com 74 pacientes, foi observado que a ocorrência de óbitos foi acima da média descrita na literatura, com incidência de 60\%.

A terapia nutricional enteral é considerada como um dos principais tratamentos utilizados no manejo do paciente crítico, visto que ele é caracterizado por alterações em seu metabolismo, através de cascatas de reações que levam ao maior risco de desnutrição. A administração precoce e adequada da terapia nutricional enteral tem se mostrado benéfica, no sentido de redução da morbimortalidade e diminuição dos casos de infecção (PINHEIRO, 2011; TELLES et al., 2015). No presente estudo, houve predomínio de pacientes do sexo masculino e com faixa etária inferior a 50 anos. Em uma pesquisa realizada no período de julho a dezembro de 2015, em uma UTI geral e Cardiológica, de um hospital Universitário no Maranhão, com 53 pacientes recebendo nutrição enteral exclusiva, verificou-se uma população do sexo masculino, mas com idade entre 61 a 80 anos, com média de permanência na unidade intensiva de 21,1 dias (ROCHA et al., 2018).

Já no estudo realizado com 25 pacientes, que estavam em TNE exclusiva, em uma UTI de um hospital de ensino do interior do Rio Grande do Sul, no período de setembro a dezembro de 2017, encontrou que os pacientes tinham idade superior a 60 anos, eram do sexo feminino e o tempo médio de internação hospitalar foi de 8,52 $\pm 1,53$ dias (FISCHER et al., 2018).
Foi encontrado em um estudo descritivo, de caráter retrospectivo e abordagem quantitativa, realizado em um Hospital Geral do Vale do Paraíba, onde foram analisados prontuários de pacientes internados na UTI, que receberam NE, no período de 2010 a 2011, que houve maior incidência de pacientes jovens (18 a 30 anos) e do sexo masculino. Esses dados podem ser explicados levando em conta o alto índice de traumas, dos casos encontrados na UTI, decorrentes na maioria das vezes, de acidentes automobilísticos (TELLES et al., 2015). Em outro estudo, de coorte prospectiva, realizado com pacientes de UTI, na cidade de Rio Branco, Acre, no período de fevereiro a novembro de 2017, acompanhados por 7 dias, verificou que os pacientes estudados eram do sexo masculino $(63,8 \%)$ tinham idade $<60$ anos $(73,8 \%)$ e apresentaram diagnóstico de trauma (49,2\%) (RITTER et al., 2019).

A oferta da nutrição precoce esteve de acordo com o que é preconizado, sendo iniciada dentro de 24 a 48 horas da admissão hospitalar (REINTAM BLASER et al., 2017; SINGER et al., 2019). Também esteve de acordo com as recomendações, o estudo do tipo série de casos realizado com amostra de 45 pacientes internados em UTI do Instituto de Medicina Integral Professor Fernando Figueira (IMIP), entre março e juIho de 2011, onde identificou o início da TNE precoce em $93,3 \%$ dos pacientes, resultados estes acima do encontrado na presente pesquisa (LINS; DIAS, 2015).

A inadequação calórica predominou nos pacientes com idade superior a 50 anos, ressaltando que a meta de proteína não foi atingida nos pacientes avaliados. Um estudo de coorte prospectivo, com 92 pacientes, internados em uma UTI do Hospital de Clínicas de Porto Alegre, no período de março a agosto de 2012 verificou que $63 \%$ dos pacientes receberam nutrição enteral precoce, porém apenas cerca de $50 \%$ atingiu as metas calóricas e proteicas no 3 ㅇ dia da in- 
ternação na UTI, ao passo que esse percentual foi reduzido no 7o dia, ficando internados na UTI durante 15,72 $\pm 10,80$ dias (PASINATO et al., 2013).

Também foi observado, que os pacientes com inadequação proteica, ingerindo menos que $1,5 \mathrm{~g}$ de proteína por kg de peso, no 3ㅇ e 70 dia de internação, foram a óbito. Os Guidelines Enteral Nutrition da Sociedade Europeia de Nutrição Enteral e Parenteral (ESPEN, European Society for Parenteral and Enteral Nutrition) e Sociedade Americana de Nutrição Enteral e Parenteral (ASPEN, American Society for Parenteral and Enteral Nutrition) recomendam que as necessidades energéticas devem ser atendidas entre o terceiro e sétimo dia de terapia nutricional enteral (MCCLAVE et al., 2016; REINTAM BLASER et al., 2017; SINGER et al., 2019).

Atingir a meta calórico-proteica é considerado um desafio para a terapia nutricional, visto que inúmeros fatores podem interferir para o fracasso no cumprimento das metas traçadas (MCCLAVE et al., 2016; SINGER et al., 2019). Semelhante ao resultado encontrado nesta pesquisa, o estudo realizado com 564 pacientes com TNE exclusiva, nas UTI's clínicas e cirúrgica do Hospital das Clínicas da Universidade Federal de Goiás, verificou que a inadequação calórica e proteica foi de $55,26 \%$ e $68,42 \%$, respectivamente. Já no estudo realizado na UTI de um hospital universitário do Rio de Janeiro, com 74 pacientes, o déficit energético médio encontrado foi de $133 \mathrm{kcal} / \mathrm{dia}$, enquanto que a inadequação proteica gerou déficits diários médios de 20,1 g (PEREIRA; WADY; VELARDE, 2016; SANTANA et al., 2016).

Diferente dos resultados encontrados na presente pesquisa, um estudo de coorte prospectiva, realizado com pacientes de UTI, na cidade de Rio Branco, Acre, no período de fevereiro a novembro de 2017, acompanhados por 7 dias, verificou que houve adequação calórica-proteica $>80 \%$ em $70,0 \%$ dos pacientes [(RITTER et al., 2019).

Assim como encontrado nesta pesquisa, o estudo realizado com 564 pacientes acompanhados diariamente durante a internação em unidade de terapia intensiva geral do Hospital das Clínicas da Faculdade de Medicina de Botucatu, por 2 anos consecutivos (de maio de 2008 a maio de 2010), divididos em com e sem injúria renal aguda adquirida, encontrou que os pacientes que desenvolveram LRA eram do sexo masculino $(55,7 \%)$ e nos pacientes com LRA houve uma adequação calórica no 3 o dia de internação, estando de acordo com o que é preconizado pela literatura (PONCE et al., 2011).
No presente estudo, foi observado que até o 30 dia de internação, dos pacientes que evoluíram com LRA, houveram intercorrências para a não infusão do volume de dieta enteral prescrita. Em um estudo observacional prospectivo, realizado com pacientes admitidos em uma UTI, recebendo TNE exclusiva, durante 53 dias, bem como infusão da dieta por meio de sistema fechado, infusão contínua (22 horas/dia), verificou que houve interrupção na infusão da dieta em 40,6\% dos pacientes estudados. Já em outro estudo longitudinal prospectivo, realizado nas enfermarias clínicas de um hospital público da cidade de Salvador (Bahia), entre setembro de 2013 e maio de 2014, com 51 pacientes em uso de TNE exclusiva, concluiu que cerca de $40,0 \%$ do volume de dieta enteral prescrito não foram administrados (SANTOS, 2017).

Durante a TNE podem ocorrer condições que interferem na administração do volume da fórmula enteral prescrito pelo nutricionista, o que pode causar uma suspensão temporária, e assim contribuir para o declínio do estado nutricional dos pacientes. Estas condições incluem o jejum para realização de exames e procedimentos, as alterações gastrointestinais, como vômitos, diarreia e distensão abdominal, a remoção da sonda e a instabilidade clínica do paciente (FISCHER et al., 2018).

No estudo realizado em uma UTI de Rio Branco, Acre, já mencionado acima, foi observado que os fatores de risco para inadequação proteico-calórica foram as interrupções da nutrição, especialmente por motivo de vômito/resíduo gástrico, jejum para procedimento de extubação, exames e procedimentos cirúrgicos (RITTER et al., 2019).

Outro estudo, de caráter prospectivo e observacional, realizado na UTI adulto de um hospital universitário de São Paulo, realizada entre os anos de 2010 a 2012, com 93 pacientes, observou que, em média, $81,6 \%$ do volume de nutrição enteral prescrito foi infundido (RIBEIRO et al., 2014).

Diferente dos resultados encontrados na pesquisa realizada em hospital público da cidade de Salvador, anteriormente citado, que mais da metade dos pacientes recebeu volume de dieta enteral inferior ao prescrito, sendo que um percentual considerável desses recebeu volume considerado baixo ou muito baixo, o que pode acarretar em piora do estado nutricional para o paciente em uso da TNE. Utilizar fórmulas enterais com reduzido teor calórico e proteico $(1,2 \mathrm{kcal} / \mathrm{ml}$ e $50 \mathrm{~g}$ de proteína/1.000 ml) pode ter colaborado com esses (resultados, uma vez que aquelas 
com teores elevados favorecem o alcance da meta nutricional em menor tempo (RIBEIRO et al., 2014; SANTOS, 2017).

Apesar da comprovação científica dos benefícios promovidos ao paciente quando se atinge as recomendações de calorias e proteínas, a UTI estudada não esteve de acordo com as preconizações. Isto pode ser explicado pelo fato de que a unidade é pública, depende de processos licitatórios anuais para a aquisição dos insumos enterais interferindo, na maioria das vezes, no fornecimento de dietas hipercalóricas e hiperproteicas.

Além do mais, vale ressaltar que as condições clínicas, a gravidade de cada paciente, a realização de procedimentos e as intercorrências são fatores que contribuem diretamente para a não adequação nutricional.

Destaca-se como limitações deste estudo a ausência de equipamentos que pudessem fazer a aferição de medidas antropométricas mais específicas, ausência de dados laboratoriais que pudessem identificar o perfil nutricional do paciente, assim como a falta de rotina de alguns exames, que são colhidos esporadicamente, como o de albumina, por exemplo, além da limitação no fornecimento de produtos enterais, devido aos processos licitatórios burocráticos.

\section{CONCLUSÃO}

A inadequação do suporte nutricional foi considerada alta, impactando diretamente no estado de saúde do paciente crítico. Contudo, observa-se que o início da TNE ocorreu na maioria dos pacientes, estando de acordo com as diretrizes, fator positivo que reflete também na qualidade da assistência ofertada ao paciente. Na ocorrência de LRA, houve maior inadequação no valor energético, bem como maior incidência de intercorrências para a não infusão da dieta até o 3o dia quando comparado aos pacientes sem LRA.

É necessário que haja uma revisão sobre os processos de aquisição dos produtos enterais, reduzindo a burocracia e melhorando a qualidade dos insumos nutricionais. Tais ações favorecerão o atendimento das necessidades dos pacientes, das metas traçadas no plano terapêutico, melhorando a qualidade da terapia nutricional ofertada e, consequentemente, contribuindo para um melhor desfecho ao paciente em estado crítico.

\section{REFERÊNCIAS}

BERBEL, M. N. et al. Nutritional parameters are associated with mortality in acute kidney injury. Clinics, v. 69, n. 7, p. 476-482, July 2014.

DHALIWAL, R. et al. The Canadian critical care nutrition guidelines in 2013: an update on current recommendations and implementation strategies. Nutrition in Clinical Practice: Official Publication of the American Society for Parenteral and Enteral Nutrition, v. 29, n. 1, p. 29-43, Feb. 2014.

FISCHER, M. D. Q. et al. Adequação calórico-proteica de pacientes internados em uma unidade de terapia intensiva. Revista de Epidemiologia e Controle de Infecção, v. 8, n. 3, p. 248-252, 6 jul. 2018.

HOSTE, E. A. J. et al. Epidemiology of acute kidney injury in critically ill patients: the multinational AKI-EPI study. Intensive Care Medicine, v. 41, n. 8, p. 1.411-1.423, Aug. 2015.

KELLUM, J. A. Diagnostic Criteria for Acute Kidney Injury. Critical Care Clinics, v. 31, n. 4, p. 621-632, Oct. 2015.

KIDNEY INTERNATIONAL SUPPLEMENTS. Kidney International Supplements, v. 2, n. 1, p. 1, Mar. 2012.

KIRSZTAJN, G. M. et al. Fast Reading of the KDIGO 2012: Guidelines for evaluation and management of chronic kidney disease in clinical practice. Jornal Brasileiro de Nefrologia, v. 36, n. 1, p. 63-73, 2014.

LINS, N. F.; DIAS, C. de A. Adequação da terapia nutricional enteral em pacientes críticos de um centro de referência em Pernambuco. Revista Brasileira de Nutrição Clinica, v. 30, n. 1, p. 76-81, 2015.

MCCLAVE, S. A. et al. Guidelines for the Provision and Assessment of Nutrition Support Therapy in the Adult Critically III Patient. Journal of Parenteral and Enteral Nutrition, v. 40, n. 2, p. 159-211, 2016.

MOHSENIN, V. Practical approach to detection and management of acute kidney injury in critically ill patient. Journal of Intensive Care, v. 5, n. 1, p. 57, 16 Sept. 2017.

PASINATO, V. F. et al. Terapia nutricional enteral em pacientes sépticos na unidade de terapia intensiva: adequação às diretrizes nutricionais para pacientes críticos. Revista Brasileira de Terapia Intensiva, v. 25, n. 1, p. 17-24, mar. 2013.

PATEL, J. J. et al. Protein Requirements for Critically III Patients With Renal and Liver Failure. Nutrition in Clinical Practice: Official Publication of the American Society for Parenteral and Enteral Nutrition, v. 32, n. 1_suppl, p. 101S-111S, Apr. 2017.

PEREIRA, D. J.; WADY, M. T. B.; VELARDE, L. G. C. Adequação energética e proteica de pacientes em terapia nutricional enteral internados em uma Unidade de Terapia Intensiva. Braspen Journal, v. 31, n. 3, p. 219-225, 2016.

PINHEIRO, M. N. A. Terapia nutricional em UTI. 2011. $30 \mathrm{f}$. Trabalho de Conclusão de Curso. Associação de Medicina Intensiva Brasileira, Cuiabá, MT, 2011.

PONCE, D. et al. Acute kidney injury in intensive care unit patients: a prospective study on incidence, risk factors and. Revista Brasileira de Terapia Intensiva, v. 23, n. 3, p. 321326, set. 2011. 
REINTAM BLASER, A. et al. Early enteral nutrition in critically ill patients: ESICM clinical practice guidelines. Intensive Care Medicine, v. 43, n. 3, p. 380-398, 2017.

RIBEIRO, L. M. K. et al. Adequacy of energy and protein balance of enteral nutrition in intensive care: what are the limiting factors? Revista Brasileira de Terapia Intensiva, v. 26, n. 2, 2014.

RITTER, C. G. et al. Risk factors for protein-caloric inadequacy in patients in an intensive care unit. Revista Brasileira de Terapia Intensiva, v. 31, n. 4, 2019.

ROCHA, A. DE J. S. C. et al. Causas de interrupção de nutrição enteral em Unidades de Terapia Intensiva/Causes of interruption of enteral nutrition in Intensive Therapy Units. Revista de Pesquisa em Saúde, v. 18, n. 1, 9 fev. 2018.

SANTANA, M. DE M. A. et al. Inadequação calórica e proteica e fatores associados em pacientes graves. Revista de Nutrição, v. 29, n. 5, p. 645-654, out. 2016.

SANTOS, A. L. Terapia nutricional enteral: relação entre percentual de dieta prescrito e administrado e intercorrências associadas em hospital público de Salvador, BA. Braspen Journal, v. 33, n. 1, p. 58-63, 2017.

SINGER, P. et al. Espen guideline on clinical nutrition in the intensive care unit. Clinical Nutrition, v. 38, n. 1, p. 48-79, Feb. 2019.

SINGER, P.; COHEN, J. How could we make nutrition in the intensive care unit simple? Revista Brasileira de Terapia Intensiva, v. 28, n. 4, 2016.

TEJERA, D. et al. Epidemiology of acute kidney injury and chronic kidney disease in the intensive care unit. Revista Brasileira de Terapia Intensiva, v. 29, n. 4, 2017.

TELLES, J. L. H. et al. Nutrição enteral: complicações gastrointestinais em pacientes de uma unidade de terapia intensiva. Revista Recien - Revista Científica de Enfermagem, v. 5, n. 13, p. 5-11, 5 abr. 2015.

TOLEDO, D.; GONÇALVES, R. C.; CASTRO, M. Meta proteica versus disfunção renal na Unidade de Terapia Intensiva. Braspen Journal, v. 31, n. 4, p. 367-370, 2016.

ZHANG, H.; ZHANG, X.; DONG, L. Nutritional status plays a crucial role in the mortality of critically ill patients with acute renal failure. Journal of Investigative Medicine, v. 66, n. 2, p. 309-318, Feb. 2018. 